\title{
The ONR-602 Experiment and Investigation of Particle Precipitation Near the Equator
}

\author{
M. A. MIAH \\ Space and Environment Studies Laboratory, Department of Mathematical Sciences, \\ University of Arkansas at Pine Bluff, Pine Bluff, AR 71601, U.S.A.
}

(Received June 26, 1990; Revised January 18, 1991)

\begin{abstract}
The ONR-602 experiment on board the S81-1 US Air Force mission was used, as one of its objectives, to investigate the global precipitation of radiation belt particles at low altitude. The experiment consisted of two particle telescopes--the main telescope and the monitor telescope. The main telescope performed Pulse-Height Analysis (PHA) on a priority basis set by the triggering of specific logical combinations of detectors to determine the charge, mass and energy of events, and returned detector coincidence and singles counting rates. Detector coincidence counting rates were formed by various logical combinations of the detectors, and singles counting rates were simply individual detector's counting rates. The monitor telescope had a fully depleted Si detector, and was designed to return only high counting rates of protons $(0.6-9 \mathrm{MeV})$, alpha particles $(0.8-4.5 \mathrm{MeV} / \mathrm{n})$, and $Z>2$ particles $(1.1-11 \mathrm{MeV} / \mathrm{n})$. The three rates were corresponding to the three discriminator thresholds $-M L(0.36 \mathrm{MeV}), M M(2.80 \mathrm{MeV})$, and $M H(10.50$ $\mathrm{MeV}$ ) for the pulse height analyzer of the single Si detector. The monitor had the peak efficiency of detecting particles of $\sim 90^{\circ}$ pitch angle near the equator, and due to the wide opening angle of $75^{\circ}$ it could detect quasitrapped particles of all pitch angles satisfying the energy thresholds in the altitude of interest. The high energy cosmic ray background count by the monitor was very insignificant. The procedures of handling data for the equatorial zone $\left( \pm 30^{\circ}\right.$ geomagnetic latitude) has been presented. Careful separation of particle precipitation data during geomagnetic average condition for the equatorial zone from other zones, and subsequent analysis show that $M L$ rates were almost entirely due to protons.
\end{abstract}

\section{Introduction}

The planet Earth is surrounded by two huge belts of radiation. Radiation belt particles are precipitated and lost into the atmosphere. The charge exchange process which is believed to be effective, works in the way that an energetic ion makes an interaction with the exospheric thermal hydrogen in which the ion is converted to an energetic neutral by electron capture. A certain fraction of this neutral is directed toward the Earth, and at low thermospheric altitudes, the neutrals are stripped off their electrons and produce the ions again. These ions are then trapped in the geomagnetic field forming a quasitrapped particle zone. Intensity of particle precipitation depends upon geomagnetic condition-lower energy particles have higher enhancement in intensity than higher energy particles.

Loss of magnetospheric ions and precipitation occur globally in four different zones. These are the equatorial zone, the low-latitude zone, the mid-latitude zone, and the 
auroral zone. This division was made by VOSS and SMITH (1980) based on the observational results for low energy protons and electrons.

Charged particle radiation in space is characterized by its composition, energy spectrum, intensity, anisotropy, and each event is completely specified by measuring parameters: charge, kinetic energy, and arrival direction. Intensity is determined by collecting events over selected time intervals or by recording counting rates from one or more detectors. A large variety of techniques have been employed to investigate one or several of the above parameters. The detection techniques produce signals that depend upon more than a single property of the incident particle, usually a function of the charge, mass and energy. This leads to the use of multiple detectors of the same or different types combined into telescope systems whose purpose is to measure sufficient parameters to characterize the event. Although the use of telescopes complicates the needed electronic circuitry and the data reduction, it provides the analytical power necessary to study space radiation in detail.

Particle precipitation at the equator has previously been studied by MORITZ (1972), HOVESTADT et al. (1972), MIZERA and BlAKE (1973), and SCHOlER et al. (1975) above $\sim 450 \mathrm{~km}$ in $\sim 0.012$ to $1.65 \mathrm{MeV}$. All the earlier observations were localized in space and spread over short times including prestorm, storm and poststorm periods. No observations have so far been carried out in the altitude range below $450 \mathrm{~km}$.

This paper focuses on the Phoenix-1 (ONR-602) instrument and the S81-1 mission, and the details of the reduction procedure of data accumulated over a period of nearly seven months (May through November of 1982) below $300 \mathrm{~km}$ altitude level. The details of the results of the data analysis and their geophysical interpretation are provided in a second paper of this issue of the journal.

\section{The S81-1 Mission and Its Objectives}

The U.S. Air Force S81-1 Mission was a low altitude $(\sim 160-290 \mathrm{~km}$ equatorial altitude) satellite in approximately polar (orbital inclination $85.5^{\circ}$ ) circular orbit. The orbital plane was 10:30-22:30 LT and the orbital time period was 90 minutes. The lifetime of the satellite was May through November, 1982. The orbit would provide access over the polar caps, to the low energy solar flare particles, and provide some time in the low altitude parts of the radiation belts, the most interesting of which may be the region of the South Atlantic Anomaly. Such an orbit would supply opportunities to study solar particle access, cut-offs, local time effects, trapped and pseudo-trapped particles, precipitating particles and transient changes (both short and long term) in the particle population, in addition to the central analysis of the solar flare composition. Further, the multiple passes over the different global precipitation zones would offer a rich statistics to study spatial structures of different global zones and their temporal effects.

\section{The Main Telescope}

The ONR-602 experiment consisted of two particle telescopes-the main telescope (not shown in the figures) and the monitor telescope. The main telescope provides counting rate information determined by logical combinations of the various detectors, and these rates supply both an absolute normalization for the pulse height analyzed 
events and an indication of the energy spectrum of the particles above a few $\mathrm{MeV} /$ nucleon. Detector coincidence counting rates are formed by various logical combinations of the detectors. Detector singles counting rates are simply individual detector's counting rates. The main telescope data provide detailed information of flux and composition for the higher energy events. However, this work does not include the data acquired by the main telescope.

\section{The Monitor Telescope}

The data presented in this work pertain to the observation of the monitor telescope. Hereon we focus on the monitor telescope (MT) which was incorporated to record high counting rates. MT (Fig. 1(a)) had a thin solid state detector (totally depleted), enclosed in a passive shielding of $\mathrm{Mg}$ with an entrance window of $1.85 \mu \mathrm{Ti}$. The passive shielding cuts protons of energy $\leq 45 \mathrm{MeV}$. The opening angle of the telescope was $75^{\circ}$, and the look direction was 2.35 degree from the zenith. The sensitive area, geometrical factor, and detector thickness were $1.0 \mathrm{~cm}^{2}, 0.49 \mathrm{~cm}^{2}$-sr, and $40.0 \mu$ respectively.

The monitor electronics basically consisted of (i) a charge sensitive pre-amplifier (CSA), (ii) a shaping amplifier (SA) ("double" at $0.6 \mu \mathrm{sec}$ ), and (iii) a discriminator with a nominal output width of $\sim 2 \mu$ sec.

The monitor system consisted of the detector and the breadboard electronics described above. The first set of data was taken by an electronic random pulse generator (TUZZOLINO, 1978). The true rates given by the monitor was remarkably low for random pulses from this fixed amplitude source. To more closely simulate the actual conditions of random rates having many amplitudes in which the monitor system would stay in space, a second set of studies was conducted with a 3 milli-curie $\alpha$-source $\mathrm{Cm}^{244}$. The source itself is essentially monoenergetic $\left(E_{\alpha}=5.8 \mathrm{MeV}\right)$. To obtain a random flux of $\alpha$-particles distributed in energy from $\approx$ zero up to the maximum energy which a proton would deposit in the monitor detector $\left(E_{\mathrm{p} \max } \sim 1.7 \mathrm{MeV}\right)$, various absorbers were used. In addition, a series of 16 collimating "holes" were used to vary the rate of $\alpha$-particles incident on the monitor system. To obtain an absolute measure of the rate at which $\alpha$-particles were incident on the monitor system through any given hole, a standard "system" consisting of the monitor detector and an Ortec CSA-shaping amplifier was used. The shaping time constant for this standard system was $0.1 \mu \mathrm{sec}$, i.e., much faster than the monitor system electronics. The standard system counted correctly (i.e., with less than $0.7 \%$ error) up to random rates of $10^{5} \mathrm{sec}^{-1}$ as determined with the random pulse generator for a pulse amplitude of $500 \mathrm{keV}(\sim 1.5 \times$ threshold $)$. For random rates (distributed in energy up to $\sim 1.7 \mathrm{MeV}$ ) up to $4 \times 10^{4} \mathrm{sec}^{-1}$, the monitor system measures rates with an error of $<\sim 5 \%$.

\subsection{Monitor discriminators and particle response}

The monitor telescope returned three counting rates which are the rates given by the three discriminator thresholds of $0.36 \mathrm{MeV}, 2.80 \mathrm{MeV}$, and $10.50 \mathrm{MeV}$ for the pulse height analyzer of the single Si detector. In Fig. 1(b), the dotted lines are threshold values. The figure, further, shows the overlapping of particle species for the $M L$ and $M M$ rates. It is found that $M L$ can be triggered by alpha particles in the energy range $\sim 0.4-80$ $\mathrm{MeV} / \mathrm{n}$ and by $Z \geq 3$ particles $\left({ }^{12} \mathrm{C}\right)$ of energy $\geq 0.7 \mathrm{MeV} / \mathrm{n} . M M$ responds to alpha particles in the energy range $0.8-4.5 \mathrm{MeV} / \mathrm{n}$ and $Z \geq 3$ particles $\left({ }^{12} \mathrm{C}\right)$ in the energy range 


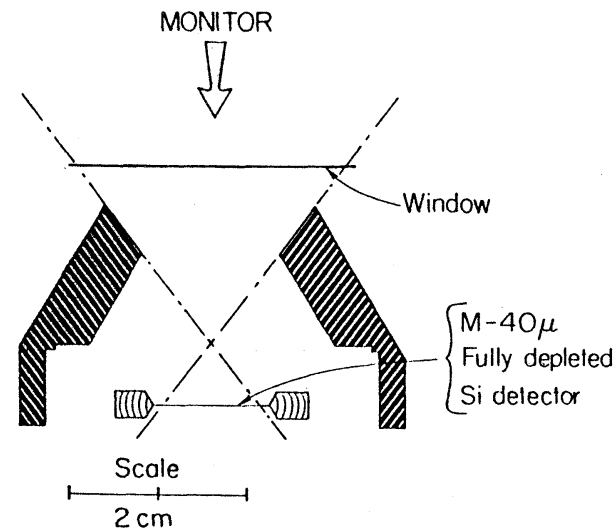

Fig. 1(a). Monitor Telescope used in the Phoenix-1 instrumentation.

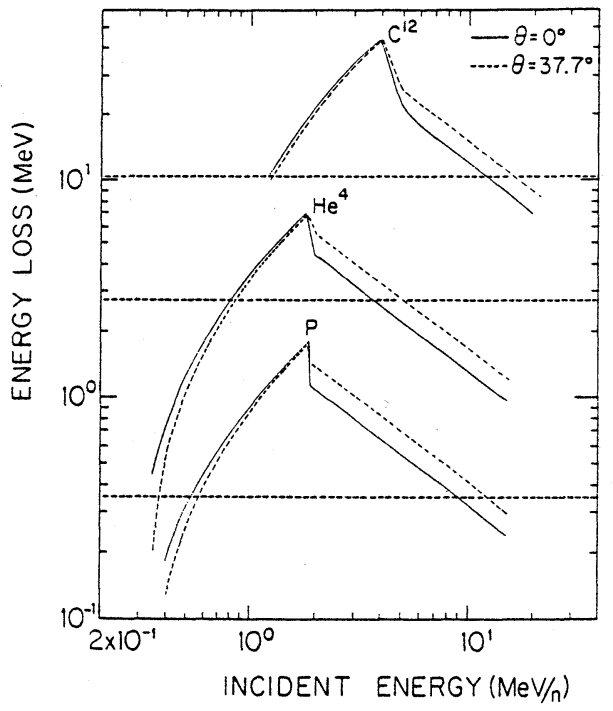

Fig. 1(b). Incident energy vs energy deposition curve.

$\sim 0.5-80 \mathrm{MeV} / \mathrm{n} . M H$ responds to $Z \geq 3$ particles in the energy range $1.2-11 \mathrm{MeV} / \mathrm{n}$ $\left({ }^{12} \mathrm{C}\right)$.

The "knee" in the energy deposition curves appear because of the very way the energy deposition was calculated. The data were taken from JANNI (1966). The given data were interpolated linearly so as to find the energy loss and range of protons in $\mathrm{Ti}$ and $\mathrm{Si}$ at intervals of $0.10 \mathrm{MeV}$. Energy of protons incident on the detector is equal to the incident energy minus the energy lost in the window material. At normal incidence, up to $\sim 0.2 \mathrm{MeV}$ protons are absorbed in the window, and at $\theta=37.7^{\circ}$ incidence, protons of $\sim 0.3 \mathrm{MeV}$ are absorbed in the window. Protons of incident energy up to $\sim 1.8 \mathrm{MeV}$ (in which case they carry $\sim 1.72 \mathrm{MeV}$ and $\sim 1.70 \mathrm{MeV}$ for $\theta=0^{\circ}$ and $\theta=37.7^{\circ}$, respectively 
because of $0.08 \mathrm{MeV}$ energy loss for $\theta=0^{\circ}$ and $0.10 \mathrm{MeV}$ energy loss for $\theta=37.7^{\circ}$ in the window) are absorbed in the detector. The energy loss figures were obtained by multiplying the energy loss per unit length by the range in the material at the given energy, if the range in the material was smaller than the path length in the detector. On the other hand, if the range in the material was greater than the path length in the detector, the energy loss per unit length was multiplied by the path length in the detector. The "knee" in the energy loss curves appear because of the sudden drop in energy deposition associated with a range longer than the path length in the detector. At the turning point, a proton of $1.8 \mathrm{MeV}$ can deposit $1.72 \mathrm{MeV}$ at normal incidence and 1.70 $\mathrm{MeV}$ at oblique $\left(\theta=37.7^{\circ}\right)$ incidence, whereas at the "knee" position a proton of 1.85 $\mathrm{MeV}$, because of its range greater than its path length in $\mathrm{Si}$, deposits $\sim 1.11 \mathrm{MeV}$ at normal incidence and $\sim 1.40$ at oblique incidence. The curve for ${ }^{4} \mathrm{He}$ and ${ }^{12} \mathrm{C}$ were prepared from the curve for proton using the following relations:

$$
(\mathrm{d} E / \mathrm{d} x)_{\substack{z \neq 1 \\ E / A=E_{\mathrm{p}}}}=Z^{2}(\mathrm{~d} E / \mathrm{d} x)_{\substack{z=1 \\ E=E_{\mathrm{p}}}}
$$

and

$$
R_{z \neq 1}\left(E / A=E_{\mathrm{p}}\right)=A / Z^{2} R_{\mathrm{p}}\left(E_{\mathrm{p}}\right) .
$$

The other heavy particles which can trigger the $M L$ rates are shown in Table 1, along with their energy ranges. However, their abundances are very low.

Table 1.

\begin{tabular}{cc}
\hline Species & Energy range $(\mathrm{MeV} / \mathrm{n})$ \\
\hline$Z=3$ & $0.34-130$ \\
$Z=4$ & $0.40-360$ \\
$Z=5$ & $0.53-1000$ \\
\hline
\end{tabular}

\subsection{Sources of background counts}

Sources of background counts can be high energy cosmic rays (CR) and their products produced by nuclear interactions in the atmosphere. The number of $\mathrm{GeV}$ range cosmic ray particles among the dominant species, i.e., $\mathrm{H}, \mathrm{C}$, and $\mathrm{Fe}$ nuclei which can trigger the discriminators in the monitor are shown in Table 2.

The figures in Table 2 are valid for measurements near the equator, since we are interested in the peak flux of particles which would be observed near the equator, a unique position within $\pm 30^{\circ}$ latitude of the orbit.

The abundances of other CR nuclei, viz., O, F, Ne, Na, Mg, Al, Si, P, S, Ca, Co, and $\mathrm{Ni}$ can be obtained from the abundance ratios of these nuclei to $\mathrm{He}$, and $\mathrm{Fe}$, which are less than a few tenths. So, the $M L$ rate is immune from any $\mathrm{GeV}$ range CR nuclei.

\subsection{Pitch angle of particles detected}

At the dipole equator the monitor received particles of equatorial pitch angle $\sim 55^{\circ}-129^{\circ}$. A detailed calculation (MIAH et al., 1989) shows that the angle of tilt of the 
Table 2 .

\begin{tabular}{ccc}
\hline Rate & Background CR nuclei & Counts/sec \\
\hline$M L$ & $\mathrm{H}$ & $1.37 \times 10^{-4}$ \\
& $\mathrm{He}$ & $2.63 \times 10^{-4}$ \\
& $\mathrm{C}$ & $2.62 \times 10^{-4}$ \\
& $\mathrm{Fe}$ & $2.87 \times 10^{-5}$ \\
$M M$ & $\mathrm{He}$ & $4.50 \times 10^{-6}$ \\
& $\mathrm{C}$ & $1.06 \times 10^{-5}$ \\
& $\mathrm{Fe}$ & $2.07 \times 10^{-6}$ \\
$M H$ & $\mathrm{He}$ & $3.10 \times 10^{-7}$ \\
& $\mathrm{C}$ & $7.15 \times 10^{-7}$ \\
& $\mathrm{Fe}$ & $2.07 \times 10^{-6}$ \\
\hline
\end{tabular}

telescope axis with the local vertical direction has insignificant effect upon the pitch angle range. The satellite being in a sun-synchronized orbit (contrary to sun-sensored orbit), the given orientation has favored detection of particles of all the pitch angles which may be quasitrapped in the altitude range of the mission. At $\sim 150 \mathrm{~km}$ altitude, the pitch angle range of particles at the equator is $\sim 90 \pm 10^{\circ}$, and at $\sim 775 \mathrm{~km}$, the pitch angle range is $\sim 90 \pm 35^{\circ}$. Because of the wide aperture, the particle flux measured by the monitor telescope is not unidirectional, and is rather omnidirectional.

\subsection{Efficiency of monitor telescope}

The calculation of efficiency function for the pitch angle $\alpha$, defined to be the fraction of the associated space angle intercepted by the telescape cone, duly weighted by the perpendicular component of the exposed fractional detector area to the incoming particles of the given pitch angle. Figure 2(a) shows the intercepted portion of the telescape and pitch angle cones for an off-centered point on the detector. Figure 2(b) shows the variation of the telescope efficiency to detect particles of different pitch angles at different latitudes and longitudes. In the real magnetic field, the peak efficiency occurs for particles of $\sim 88^{\circ}$ pitch angles at the line of minimum magnetic field.

\section{Data Reduction}

\subsection{Monitor data coverage}

The ONR-602 data has undergone several stages of reduction/processing. Lockheed Palo Alto Laboratory undertook the task of digitizing the raw data from remote tracking stations, and of making it available to the experimenters. The University of Chicago was in charge of processing the ONR-602 data at the preliminary stage, eliminating the bad readouts, and subdividing the dataset into CHART magnetic tapes and COALT magnetic tapes. The CHART tapes contain the ONR-602 instrument rate readouts, and COALT tapes contain pulse height analysis data. Both types of tapes were generated on the Chicago Harris Computer, a 3 byte per word machine. LSU was given copies of both kinds of tapes. At LSU, before the starting of the data analysis, algorithm were developed, tested, and applied to reformat the tape data for use in a DEC-11/23 laboratory data system, a two byte per word machine. 


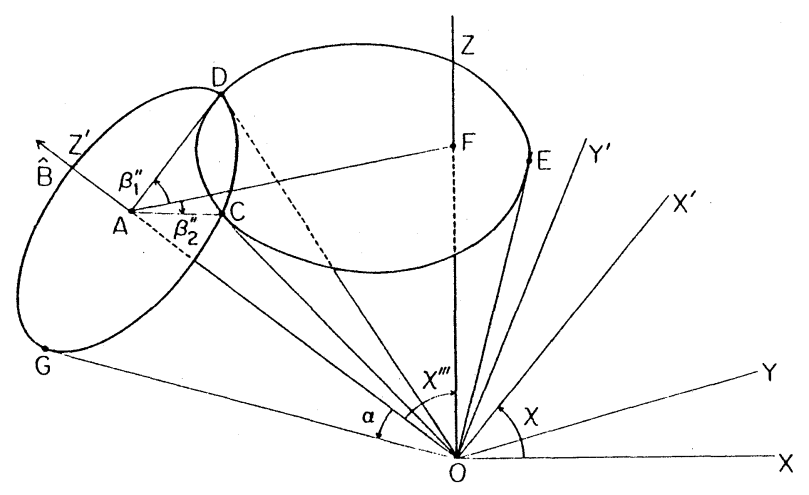

Fig. 2(a). Intersection of the pitch angle cone OCDG and the telescape cone OCDE produces the space angle OCD. The intercepted portion of the space angle is measured for all pitch angle cones at all representative points on the detector base.

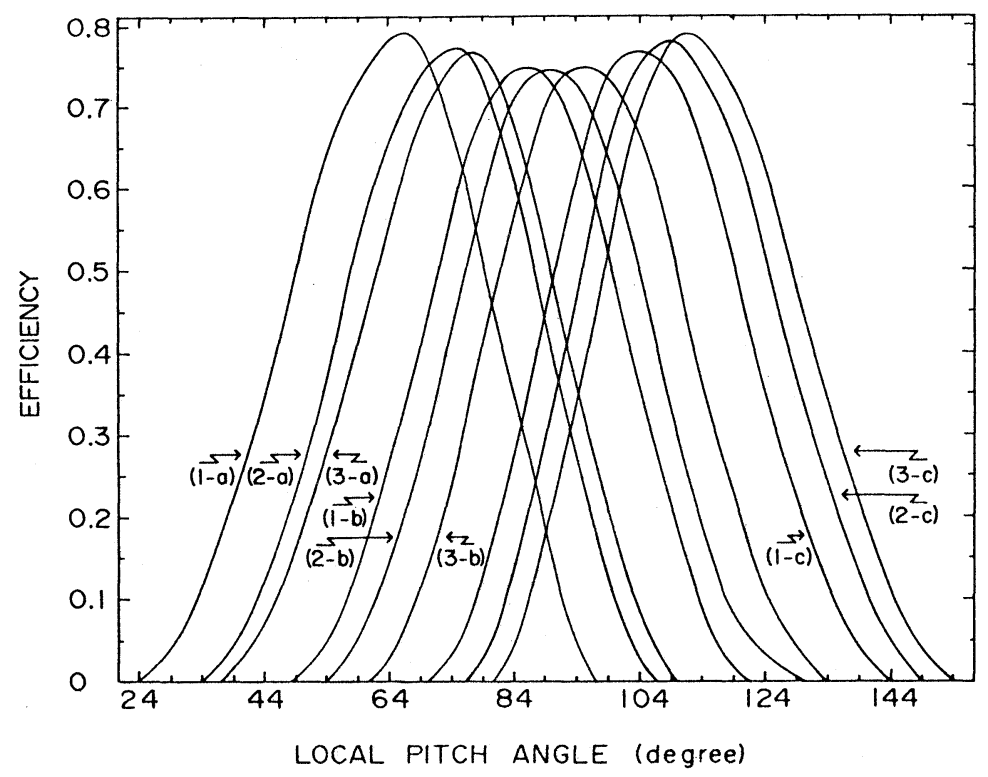

Fig. 2(b). Efficiency of the monitor telescope. b-curves are at latitudes of minimum $B$. a-curves are $-10^{\circ}$, and c-curves $+10^{\circ}$ away from latitudes of minimum $B$. Curve $(1-\mathrm{a}, 1-\mathrm{b}, 1-\mathrm{c})$ are at longitude $102^{\circ},(2-\mathrm{a}, 2-\mathrm{b}, 2-\mathrm{c})$ are at $270^{\circ}$, and $(3-\mathrm{a}, 3-\mathrm{b}, 3-\mathrm{c})$ are at $230^{\circ}$.

In nearly seven months' active lifetime, the S81-1 mission had $\sim 3000$ passes around the globe in polar orbits. These multiple passes meant statistically rich data acquisition, and offered a unique opportunity to investigate different global zones. Global data coverage plots were prepared to find the "hot spots." Figure 3(a) shows a plot of $M L$ readout. Every point on the plot indicates the instrument's physical presence and a readout (zero or nonzero) by MT. Darker parts are due to repeated passes. The gaps (except the ones near the poles) are the spots where the detector was shut-off to save 
DAIA COVERAGE

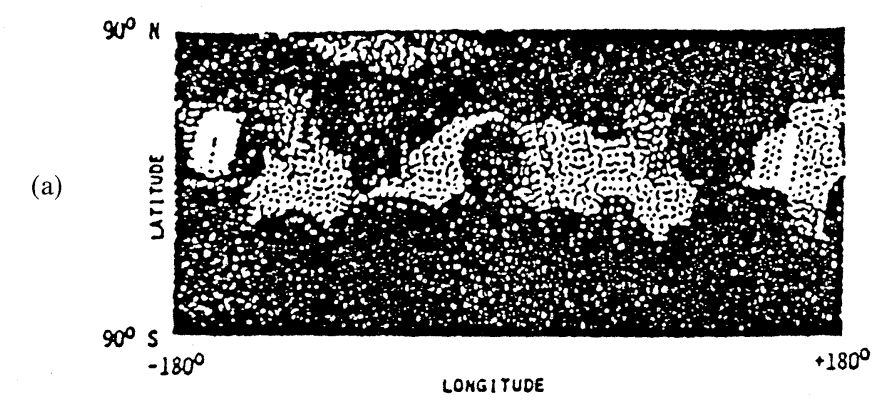

RATE $M L 2 \geqslant 5$

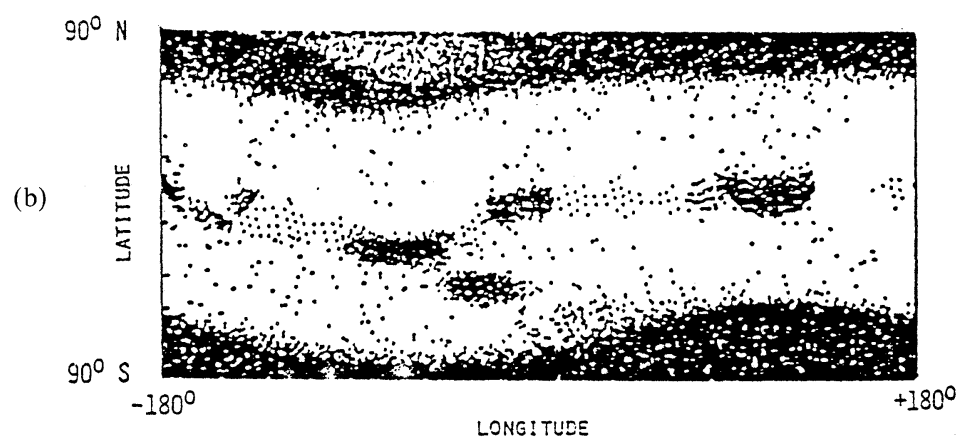

Fig. 3. Figure 3(a) represents the coverage plot. Every dark point indicates active presence of the detector in that location. Figure 3(b) is a global plot for $M L$ rate $\geq 5$. Both the plots are in geographic coordinates.

energy for data coverage at other places of interest. Figure 3(b) is a plot of $M L$ readouts $\geq 5$ which shows the equatorial zone near the middle of the figure. Every point on the plot indicates a readout by MT. Away from it and onward to the poles are the traces of particle precipitation in other global zones. The plots have been prepared in geographic coordinates. Plot of global data for different readouts are useful for locating the demarkation between the global zones.

The equatorial zone data was defined to be the data pertaining to the satellite passes over the equatorial zone which lies within $\pm 30^{\circ}$ geomagnetic latitude. This data was separated from the whole mission.

\subsection{Removal of contamination from equatorial zone data}

Principal contaminations in the raw data were the influences of the South Atlantic Anomaly (SAA) and rate spikes. The SAA (the cluster of points below the equatorial strip in Fig. 3(b)) region which is characterized by magnetic field lower than the equatorial magnetic field, and by particle flux higher than the equatorial particle flux, is 
located, approximately, between $11^{\circ} \mathrm{S}$ to $28^{\circ} \mathrm{S}$ latitude and $5^{\circ} \mathrm{W}$ to $35^{\circ} \mathrm{E}$ longitude in geomagnetic coordinates. These contaminations were carefully removed from the raw data before these were subjected to final analysis. Data gaps in the global coverage were also major problems.

\subsubsection{SAA influence}

The first problem was how to separate the SAA data from the equatorial zone data with the least possible loss of data. To solve the problem, plots of the equatorial zone data were made in $B$-L (Figs. 4(a), (b), and (c)) and geomagnetic (Figs. 5(a), (b), and (c))

$M L \geqslant I$

(a)

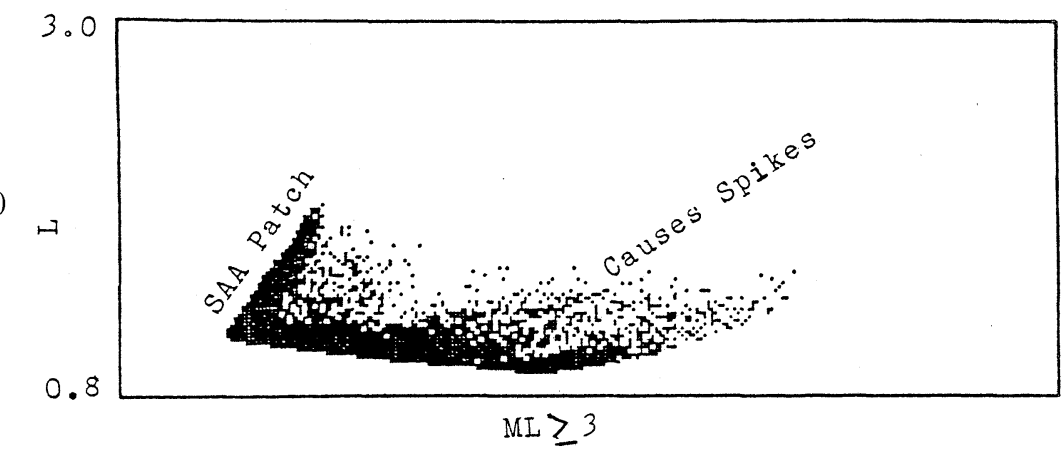

(b)

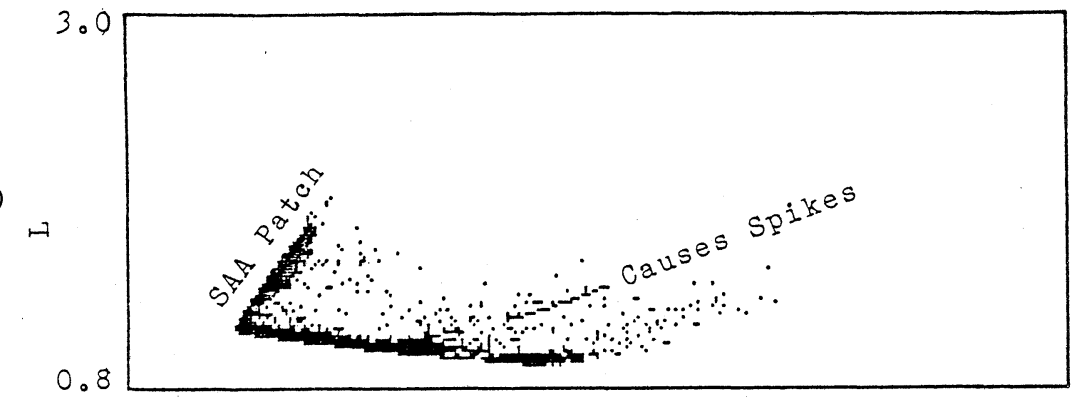

$M L \geq 5$

(c)

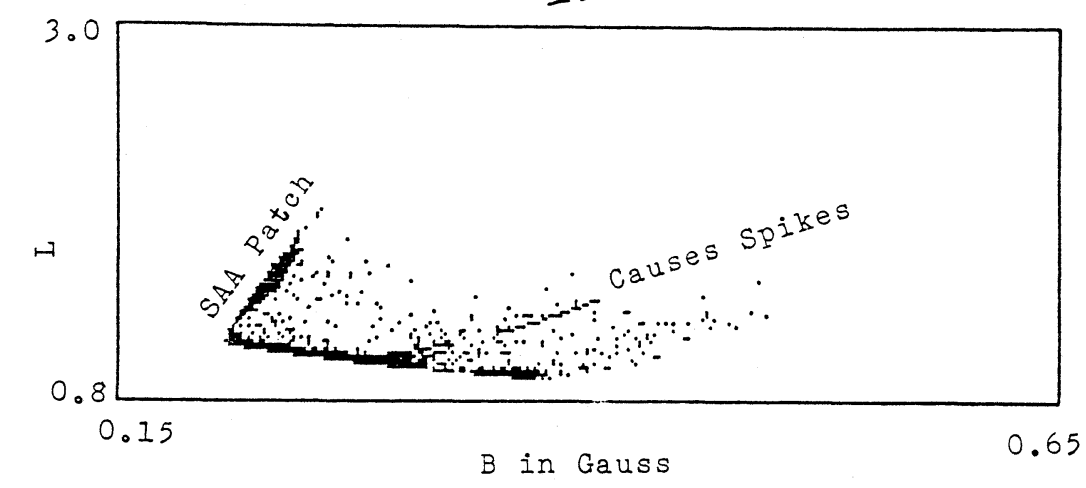

Fig. 4. Plots of the equatorial zone data in $B$ - $L$ coordinates (Figs. 4(a) for $M L \geq 1,4$ (b) for $M L \geq 3,4$ (c) for $M L \geq 5$. Locations of the contaminating SAA patch and the $M L$ rates causing spikes are indicated. The SAA part and the spikes appear more distinctly at higher threshold $M L$ rats. 

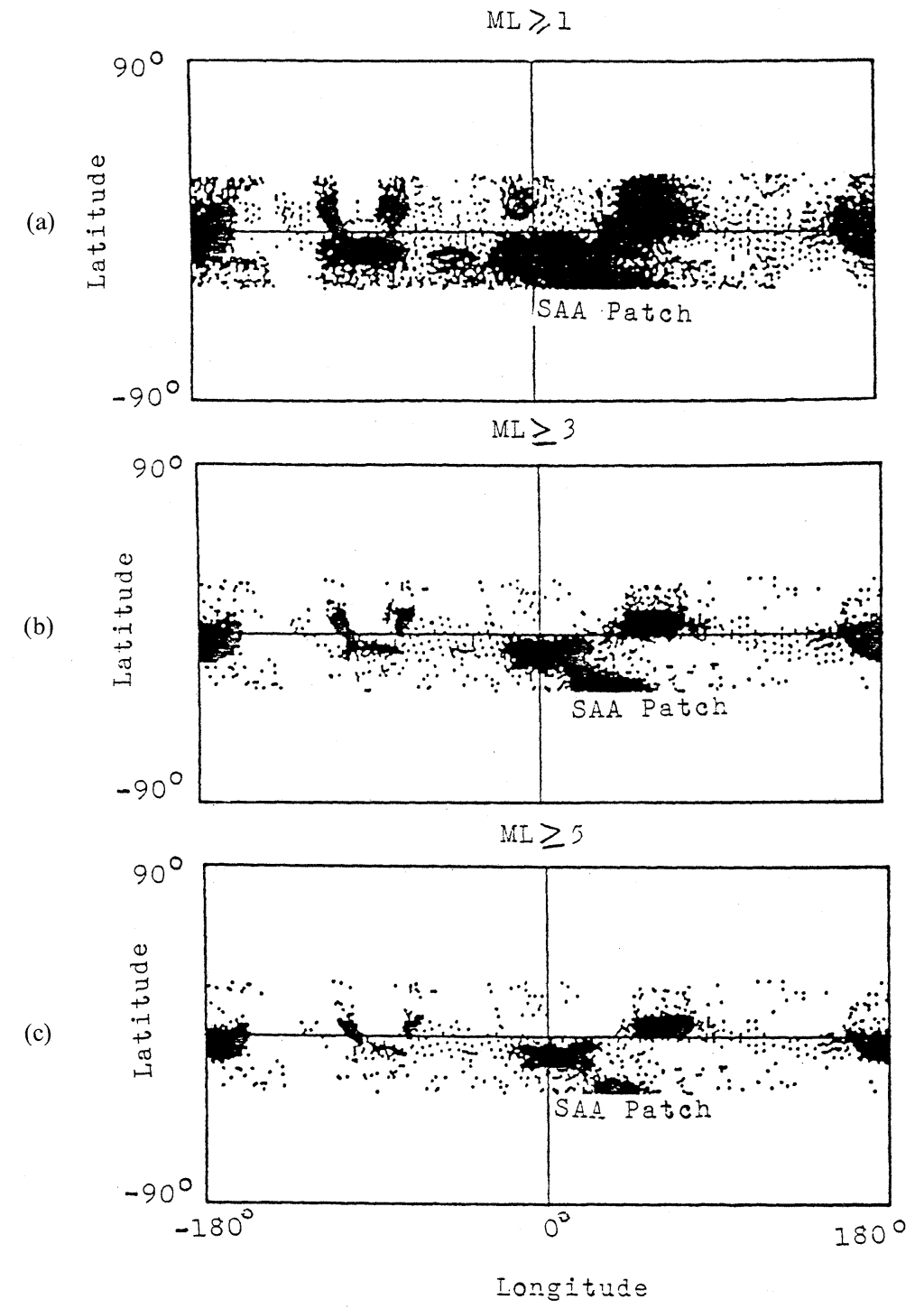

Fig. 5. Plots of the equatorial zone data in geomagnetic coordinates (Figs. 5(a) for $M L \geq 1,5$ (b) for $M L \geq 3$, 5(c) for $M L \geq 5$ ). Location of SAA patch appears distinctly at higher threshold values.

with different threshold count rates. Here again each point in a plot indicates a readout satisfying the criterion. Separation of data occurring in a certain latitude and longitude range may work at the cost of a huge chunk of data. Also it has the associated complicacy of defining SAA location of major influence in a regular or irregular shape. From a careful examination of the plots it became clear that the data points contributing SAA influence can be separated by cuts in $B-L$ values. This contaminated part was removed and fresh plots were prepared again in all the three coordinate systems, $B$ - $L$ (Figs. 6(a), 


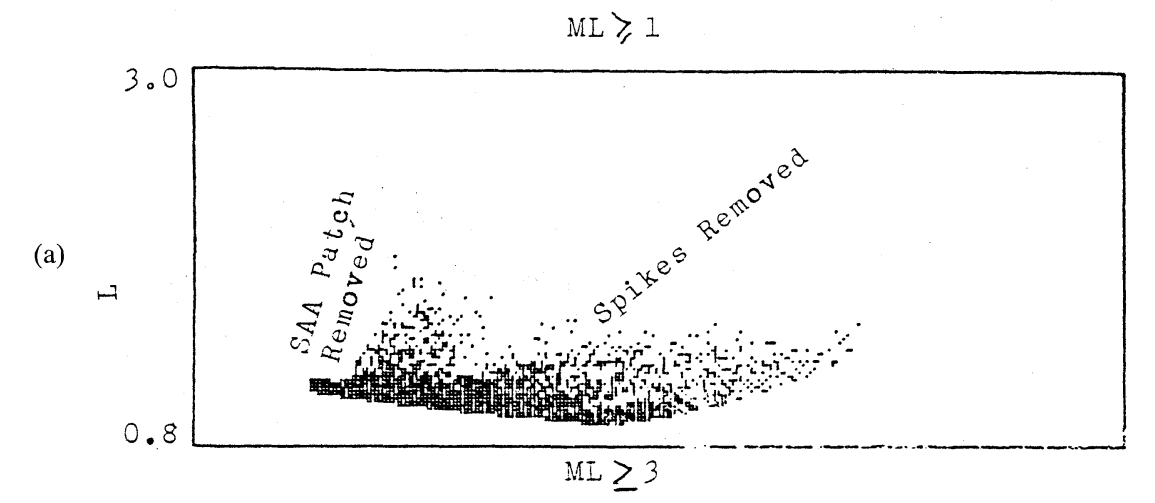

(b)
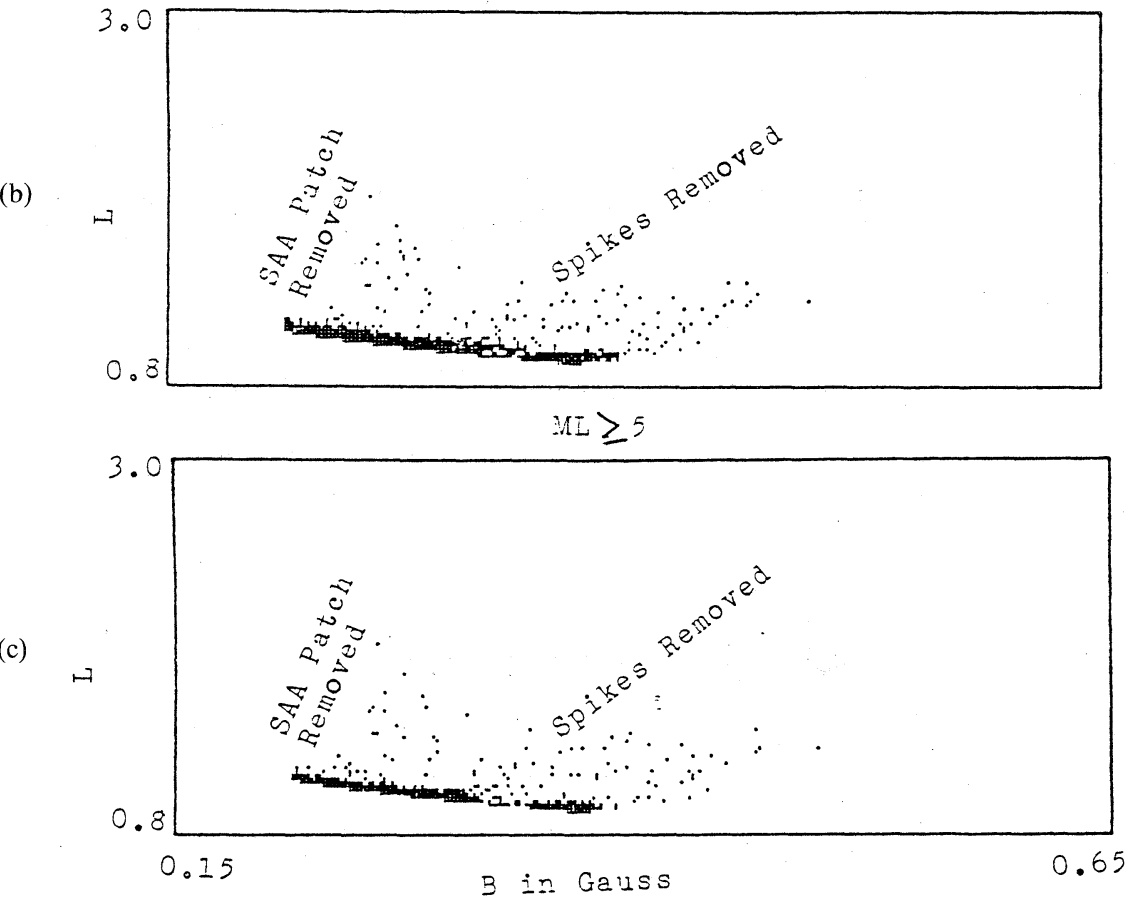

Fig. 6. Plots of cleaned data for the equatorial zone in $B-L$ coordinates. SAA patch and the spikes have been removed. Figures 6(a) for $M L \geq 1,6(\mathrm{~b})$ for $M L \geq 3$, and $6(\mathrm{c})$ for $M L \geq 5$.

(b), and (c)), and geomagnetic (Figs. 7(a), (b), and (c)) for both the equatorial zone and the contaminating SAA to check if the desired separation was performed. No separate plots for the contaminating SAA part have been shown here. This way of separation of SAA does not make the equatorial data totally free from the SAA contamination; however, this is an effective way of eliminating the major SAA influence.

\subsubsection{Rate spikes}

The data, thus cleaned of SAA contamination was containing rate spikes which were 
$M L \geqslant 1$

(a)
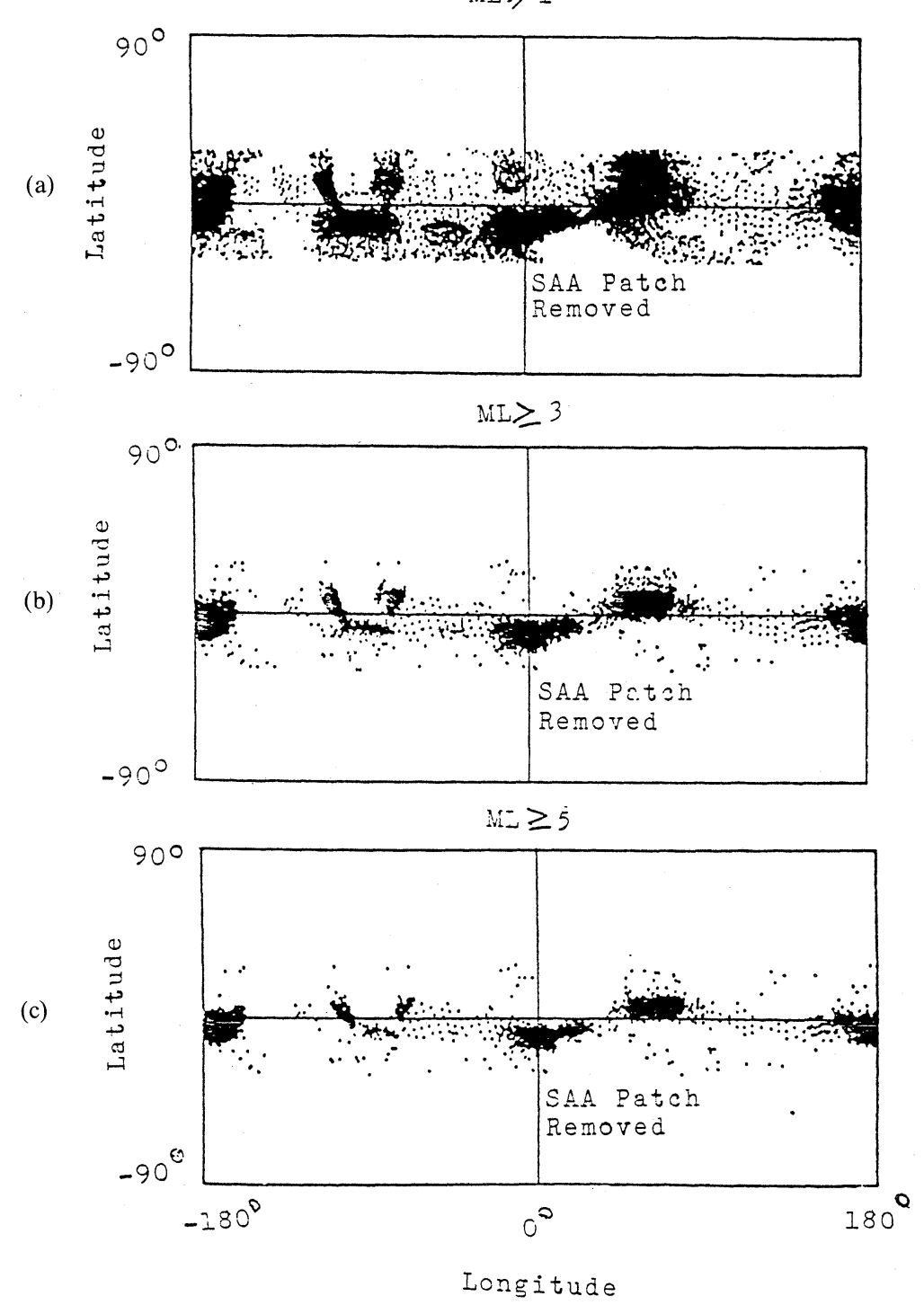

Fig. 7. Plots of cleaned data for the equatorial zone in geomagnetic coordinates. Figures 7 (a) for $M L \geq 1,7$ (b) for $M L \geq 3$ and 7(c) for $M L \geq 5$.

indicated by large overshots in the plots of count rates versus time. Their locations in different coordinates are shown in Figs. 4 through 7, and are distinctly identified in Fig. 4. To clean the data of the rate spikes, plots of $M L$ rates were made with the counting rates versus frequency of occurrence of the rate during the whole mission time (Figs. 8(a), (b), and (c)). It is found that 28 was the highest count rate with the minimum frequency. Above 28 , the frequency increases again. We do not have any apparent cause for this. Thus, to a first approximation all the subcoms (into which the magnetic tape data were 


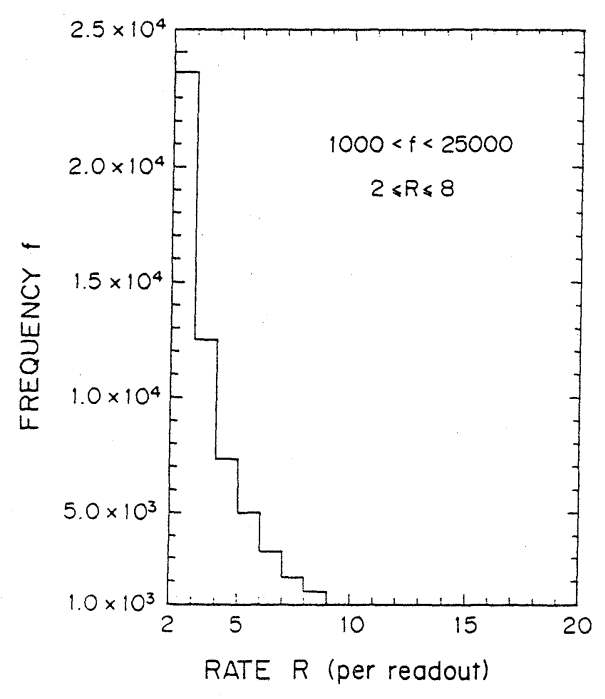

(a)

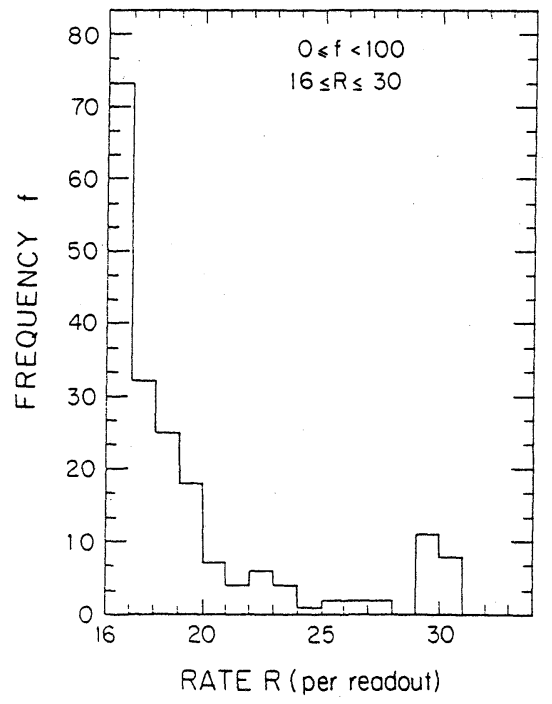

(b)

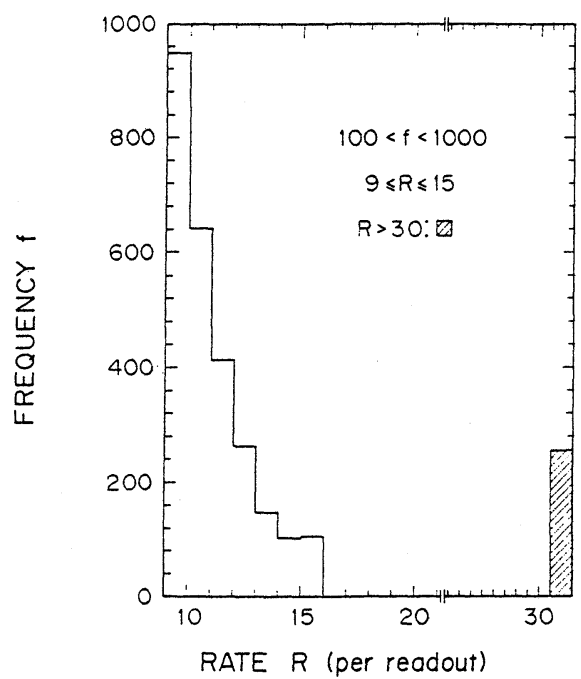

(c)

Fig. 8. Plots illustrating the different counting rates per readout versus the frequence of occurrences of rates.

structured) containing count rates more than 28 were removed. The plot shown in Figs. $6(\mathrm{a}),(\mathrm{b}),(\mathrm{c})$, and $7(\mathrm{a}),(\mathrm{b}),(\mathrm{c})$ are the plots of cleaned data in $B-L$, and geomagnetic coordinates, respectively.

\subsubsection{Geomagnetic storm influence}

Three major geomagnetic storm occurred in 1982 during the active life-time of the mission. Figure 9 shows the daily plot of the $D_{\text {st }}$ values for the period of MT data being treated in this work. The occurrences of storms are marked by the large drop of $D_{\text {st }}$ values 


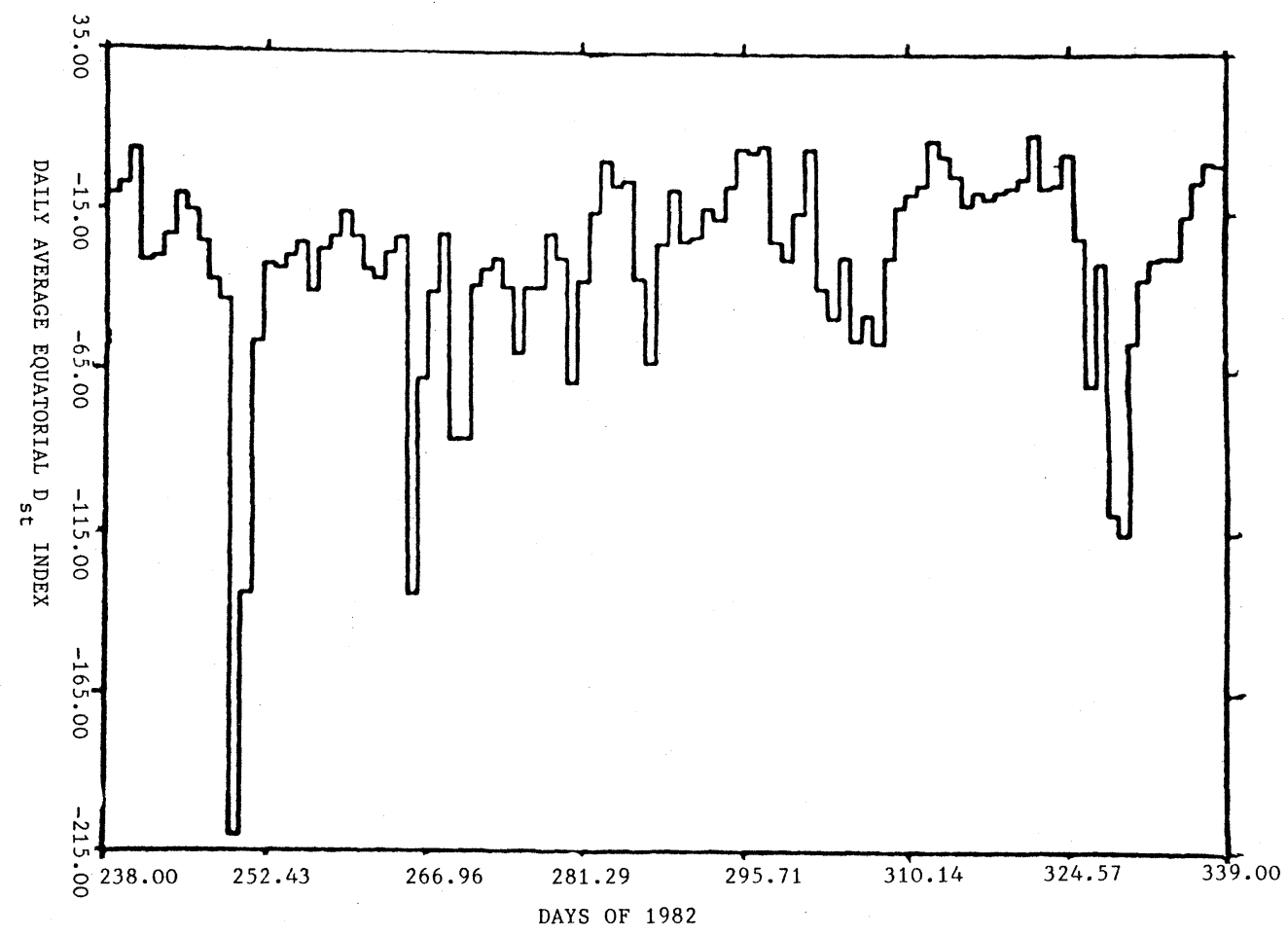

Fig. 9. Plot of daily $D_{\text {st }}$ data of 1982 for the period of data being analyzed here.

in the figure. Most of the bad quality data appeared during the stormtime. Selection of good quality data made holes in data coverage during prestorm, stormtime, and poststorm days. The data used in the analysis were, thus, pertaining to geomagnetic average condition.

\subsection{Final data}

A satellite pass over the equator within $\pm 30^{\circ}$ geomagnetic latitude has 16 subcoms of data. In the removal of bad quality data, subcom gaps were introduced in many of the satellite passes. Nothing could be done to fill up the gaps other than discarding the satellite passes for the final analysis of data. In the study it was found that only 137 passes did not have any gaps in the middle. These passes had 12 to 16 subcoms of data of which the fewer than 16 subcoms had gaps near $+30^{\circ}$ or $-30^{\circ}$, i.e., near the beginning and ending of the equatorial portions of the passes. Figure 10 shows a plot of the 54 out of the 137 passes in geomagnetic coordinates. The gap in the longitude range $0^{\circ}$ to $50^{\circ}$ arises because of the removal of the deep SAA coverage part from the equatorial coverage. Passes in this longitude range have fewer than 12 subcoms. To study the features of the low altitude proton belt, we have mainly used the data contained in the 137 passes. Only in the case of constructing global profile of peak $M L$ rates, we have included passes with 9 to 12 subcoms which did not have any gaps around the equator, to fill the part between $0^{\circ}$ to $50^{\circ}$ longitude range. 


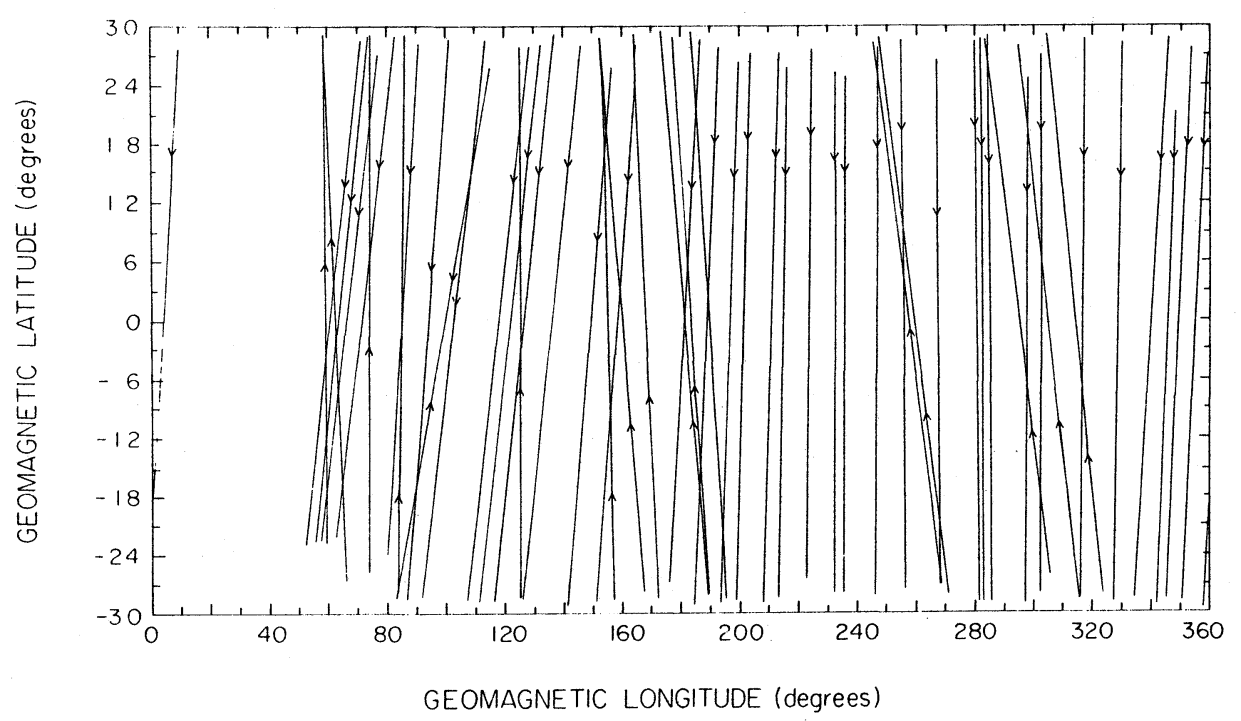

Fig. 10. A partial plot of the satellite orbits falling in the equatorial zone in geomagnetic coordinate.

\subsection{Instrumental background}

The background $M L$ rate has been taken to be the $M L$ rates detected on the outskirts of the equatorial zone. The equatorial count rates are superimposed on the background count rates which are always present. To determine the background count rates, a spot was selected away from the equatorial zone. It was bounded by the geographic latitude of $-8^{\circ}$ to $-20^{\circ}$ and geographic longitude $100^{\circ}$ to $146^{\circ}$. This zone was crossed 16 passes. From count rates per readout, the background level is found to be less than 0.01 proton per readout per degree latitude per pass.

\subsection{ML rates due to protons}

We have compared the count rates of $M L, M M$, and $M H$ in those 137 passes considered. It was found that $M M / M L \sim 10^{-3}, M H / M L \sim 10^{-4}$, and $M H / M M \sim 10^{-1}$. These ratios correspond well with the published values for the composition (SPJELDVIK and FrITZ, 1983) and indicate that $M L$ counting rates were almost entirely due to protons.

\section{Conclusion}

The combination of the main telescope, beginning analysis at a few $\mathrm{MeV} /$ nucleon, and the monitor system, giving results below $1 \mathrm{MeV} /$ nucleon, is designed to provide a versatile tool for measuring particle phenomena characterized by almost any energy spectrum. The monitor provides an indication of the presence of the particles at low energy, while the main system gives detailed flux and composition data for the higher energy events. The study of the instrument performance reveals that at the equator the monitor telescope has the peak efficiency for particles of $\sim 90^{\circ}$ pitch angles. The large opening angle of the telescope allows detection of omnidirectional flux of quasitrapped 
particles. The way of separation of the equatorial zone from the adjoining SAA seems to work well with the least loss of data is supported by the fact that our findings of the ratios of fluxes of alpha particles to protons, and heavier ions to protons agree with previous findings, whereas a significant contamination by the SAA data could alter the ratio. Further, it is because of the equatorial position in an orbit that the high energy cosmic ray background count has been very insignificant. Finally, it has been successfully demonstrated that the particle counting rates for the low energy threshold, have been almost entirely due to protons. Results of the analysis of the cleaned data and their geophysical interpretation will form a second paper.

The ONR-602 instrument was built by the Laboratory for Astrophysics and Space Research at the University of Chicago under NASA Contract NAS 2-24430, and sponsored by ONR, with launch support and telemetry coverage by the Air Force Space Test Program. The work was partly supported at Louisiana State University ONR under to Contract N-00014-83-K-0365, and partly at the University of Arkansas at Pine Bluff by AFOSR under contrast F49620-89-C-0071. The United States Government is authorized to reproduce and distribute reprints for governmental purposes notwithstanding any copyright notation hereon.

\section{REFERENCES}

Hovestadt, D., B. Hausler, and M. Scholer, Observation of energetic protons at very low altitudes near the geomagnetic equator, Phys. Rev. Lett., 28, 1340-1344, 1972.

JANNI, J. H., Calculation of energy loss, range, pathlength, straggling, multiple scattering, and the probability of inelastic nuclear collision for 0.1 to $1000 \mathrm{MeV}$ protons, Technical Report No. AFWL-TR-65-150, Kirkland Air Force Base, New Mexico, 1966.

Miah, M. A., J. W. Mitchell, and J. P. Wefel, Magnetospheric particle detection efficiency of a conical telescope, Nucl. Instr. Methods in Phys. Res., A281, 622-627, 1989.

Mizera, P. F. and J. B. Blake, Observation of ring current protons at low altitudes, J. Geophys. Res., 78, 1058-1062, 1973.

MORITZ, J., Energetic protons at low equatorial altitudes: A newly discovered radiation belt phenomenon and its explanation, Z. Geophys., 38, 701-717, 1972.

Scholer, M., D. Hovestadt, and G. Morfill, Energetic He ions from the radiation belt at low altitudes near the equator, J. Geophys. Res., 80, 80-85, 1975.

SPJELDVIK, W. N. and T. A. Fritz, Experimental determination of geomagnetically trapped energetic heavy ions, in Energetic Ion Composition in the Earth's Magnetosphere, edited by R. G. Johnson, pp. 393-396, D. Reidel, Boston, 1983.

Tuzzolino, T., Characteristics of SIRE monitor system, Laboratory for Astrophysics and Space Research, The University of Chicago, Chicago, Illinois, July 13, 1978.

Voss, H. D. and L. G. Smith, Global Zones of energetic particle precipitation, J. Atmos. Terr. Phys., 42, 227-239, 1980. 